\title{
Drug resistance in B and non-B subtypes amongst subjects recently diagnosed as primary/recent or chronic HIV-infected over the period 2013-2016: Impact on susceptibility to first-line strategies including integrase strand-transfer inhibitors
}

\author{
Samantha Andreis ${ }^{\mathrm{a}, 1}$, Monica Basso ${ }^{\mathrm{a}, 1}$, Renzo Scaggiante ${ }^{\mathrm{a}}$, Mario Cruciani ${ }^{\mathrm{b}}$, \\ Roberto Ferretto ${ }^{\mathrm{c}}$, Vinicio Manfrin ${ }^{\mathrm{d}}$, Sandro Panese ${ }^{\mathrm{e}}$, Maria Cristina Rossi $\mathrm{f}^{\mathrm{f}}$, \\ Ermenegildo Francavilla ${ }^{\mathrm{g}}$, Caterina Boldrin ${ }^{\mathrm{a}}$, Mario Alvarez ${ }^{\mathrm{a}}$, Federico Dal Bello ${ }^{\mathrm{a}}$, \\ Carlo Mengoli ${ }^{\mathrm{a}}$, Ombretta Turriziani ${ }^{\mathrm{h}}$, Loredana Sarmati ${ }^{\mathrm{i}}$, Guido Antonelli ${ }^{\mathrm{h}}$, \\ Massimo Andreoni ${ }^{\mathrm{i}}$, Giorgio Palù ${ }^{\mathrm{a}}$, Saverio Giuseppe Parisi ${ }^{\mathrm{a}, *}$ \\ a Department of Molecular Medicine, University of Padova, Padova, Italy \\ ${ }^{\mathrm{b}}$ Center of Diffusive Diseases, Verona, Italy \\ ${ }^{\mathrm{C}}$ Clinical Infectious Diseases, Hospital of Santorso, Santorso, Italy \\ d Infectious Diseases, Vicenza Hospital, Vicenza, Italy \\ e Infectious Diseases Unit, dell'Angelo Hospital, Venezia, Italy \\ f Infectious Diseases, Treviso Hospital, Italy \\ ${ }^{g}$ Infectious Diseases, Belluno Hospital, Belluno, Italy \\ ${ }^{\mathrm{h}}$ Laboratory of Virology, Department of Molecular Medicine, Istituto Pasteur Italia-Cenci Bolognetti Fondazione, Sapienza University of Rome, Rome, Italy \\ ${ }^{\mathrm{i}}$ Clinical Infectious Diseases, Tor Vergata University, Rome, Italy
}

\section{A R T I C L E I N F O}

\section{Article history:}

Received 20 February 2017

Received in revised form 30 April 2017

Accepted 3 May 2017

Available online 18 July 2017

\section{Keywords:}

Integrase strand transfer inhibitors

Transmitted drug resistance mutations

Primary-recent

Chronic HIV infection

\begin{abstract}
A B S T R A C T
Objectives: To characterize the prevalence of transmitted drug resistance mutations (TDRMs) by plasma analysis of 750 patients at the time of HIV diagnosis from January 1, 2013 to November 16, 2016 in the Veneto region (Italy), where all drugs included in the recommended first line therapies were prescribed, included integrase strand transfer inhibitors (InNSTI).

Methods: TDRMs were defined according to the Stanford HIV database algorithm.

Results: Subtype B was the most prevalent HIV clade (67.3\%). A total of 92 patients (12.3\%) were expected to be resistant to one drug at least, most with a single class mutation (60/68-88.2\% in subtype B infected subjectsand $23 / 24-95.8 \%$ in non-B subjects) and affecting mainly NNRTIs. No significant differences were observed between the prevalence rates of TDRMs involving one or more drugs, except for the presence of E138A quite only in patients with B subtype and other NNRTI in subjects with non-B infection.

The diagnosis of primary/recent infection was made in 73 patients (9.7\%): they had almost only TDRMs involving a single class. Resistance to InSTI was studied in 484 subjects (53 with primary-recent infection), one patient had $143 \mathrm{C}$ in 2016 , a total of thirteen $157 \mathrm{Q}$ mutations were detected (only one in primary/recent infection).

Conclusions: Only one major InSTI-TDRM was identified but monitoring of TDRMs should continue in the light of continuing presence of NNRTI-related mutation amongst newly diagnosed subjects, sometime impacting also to modern NNRTI drugs recommended in first-line therapy.

(c) 2017 International Society for Chemotherapy of Infection and Cancer. Published by Elsevier Ltd. All rights reserved.
\end{abstract}

\footnotetext{
* Corresponding author at: Department of Molecular Medicine, University of Padova, Via Gabelli 63, 35100 Padova, Italy. Fax: +390498272355.

E-mail address: saverio.parisi@unipd.it (S.G. Parisi).

1 These two authors equally contributed to the work.
} 


\section{Introduction}

More recent guidelines support the recommendation that all patients with chronic HIV infection with detectable plasma HIV viremia should start antiretroviral therapy (ART), regardless of CD4 + cells count: the number of naive subjects who will be treated is expected to increase in high-income countries and testing for transmitted drug resistance mutations (TDRMs) is recommended and cost-effective [1,2]. A definite and updated figure of TDRMs burden is essential to build up therapeutic algorithms but the prevalence rates differ because of the characteristics of patients studied (infection by B or non-B subtype, recent or chronic disease) and of availability of an efficient ART. Furthermore, there is an underestimation of TDRMs when they are determined on plasma samples and not on peripheral blood mononuclear cells (PBMCs): less fit strains may be archived because wild-types had a better replicative capacity in both acute and chronic patients $[3,4]$.

The reported frequency of TDRMs ranged from $9.1 \%$ to $15 \%$ in United States and it was below 10\% in Europe [5-9]. The overall prevalence in a European study (including Israel) on 4140 patients who were newly diagnosed between 2008 and 2010 was $8.3 \%$ : it was slightly higher in recently infected subjects (10.1\%) than in patients with an unknown date of infection (8.2\%) [9]. An individual-patient-level meta-analysis on 11,802 HIV-1 reverse transcriptase sequences with or without protease retrieved from 42 studies (30 countries) showed a median study-level prevalence of $9.4 \%$ : 5.6\% of TDRMs were for nucleoside reverse transcriptase inhibitors (NRTIs), 3.4\% for non-nucleoside reverse transcriptase inhibitors (NNRTIs), 1.5\% for protease inhibitors (PIs), with a subtype B prevalence of $67 \%$ [10].

With regard to primary/recent infections, TDRMs were detected in 67 of 496 cases (13.5\%) in USA: the most common major DRM (drug resistant mutation) identified were associated with NNRTIs resistance at $8.5 \%(42 / 496)$, with an increasing trend in the proportion of patients with TDR (transmitted drug resistance) and with TDR for NNRTIs throughout the study period (1996-2013) [11]. In Europe a 9.4\% prevalence was described in a Spanish cohort for the time period 2009-2012 [12], comparable to that reported in a French study (8.6\%, years 2007-2012) [13].

In Italy we previously described a TDRMs rate of $11.3 \%$ in patients with chronic subtype B infection $(6.2 \%$ in subjects with non-B subtype) and of $15.2 \%$ in subjects with primary infection ( $8.7 \%$ in patients with non-B subtype) in the period 2010-2012. The study started on 2004 and demonstrated a decline of PI resistance and of combined resistance to two or three classes of drugs during the study period [14]. The decrease of PI resistance was confirmed by Montagna et al. [15], who analysed DRMs in a cohort of Italian patients including both naive patients and subjects with virological failure who made routine controls from 2003 to 2014. Integrase strand transfer inhibitors (InSTI) were recently included in the first line regimens because of their efficacy and excellent tolerability $[1,2]$ : InSTI resistance was not found in naive patients before the drug was included in clinical practice and not even in 533 European patients tested between 2008 and 2011 [16,17]. Of note, Frange et al. [13] reported an integrase related mutation rate of $1.5 \%$ in a cohort of 1318 French patients with primary infection diagnosed in 2007-2012. This data was not confirmed in the patients of the Swiss cohort (0.1\% in the study period 2008-2014) and in Spanish patients with acute/recent infection diagnosed from May 2015 to May 2016, possibly for the low number of subjects enrolled in these two studies $[18,19]$.

Here, we characterize the prevalence of TDRMs and the circulation of non-B subtypes by plasma analysis of 750 patients at the time of HIV diagnosis from January 1, 2013 to November 16, 2016 in the Veneto region (Italy), where all drugs included in the recommended first line therapies were prescribed, included InSTI.

\section{Material and methods}

\subsection{Study design}

Adult ART-naive-infected subjects were consecutively enrolled in the study at the time of HIV infection diagnosis in seven infectious disease units located in Veneto, Italy, between January 1 , 2013 and November 16, 2016. Eligibility criteria included age $>18$ years and antiretroviral drug-naive status according to a personal interview and a review of the history of infection since the first positive serological test, which was always performed at the enrolling hospital.

The study was approved by the Ethical Committee for Clinical Experimentation, Padua Province (Ethics Committee Protocol no. 2606-12P). The subjects gave informed consent for all procedures and for the use of their blinded data for scientific evaluation and publication. This study was conducted in accordance with the Helsinki Declaration and local legislation.

Blood samples were processed within $6 \mathrm{~h}$ from collection to the Laboratory of Virology at the University Hospital of Padova (Padova, Italy) and stored until analysis.

Primary or recent HIV infection was defined by the presence of any of the following: negative or indeterminate HIV antibodies by enzyme-linked immunosorbent assay (ELISA) associated with a positive plasma HIV RNA test; or an initially negative test for HIV antibodies followed by positive serology within 18 months. The absolute CD4+ cell count, percentage of CD4+ cells and HIV-RNA plasma viral load were determined at sampling. HIV-RNA values were not reported or used for statistical analyses because the measurements during the 13 years were performed using the current commercial methods, which changed over the course of the study and exhibited a wide range of sensitivities, making the values non-comparable. The results were compared with those of the previous survey performed in 2004-2012 using the same inclusion criteria [14].

\subsection{HIV genotyping analysis}

Genotyping was performed as previously described [3]. All resistant strains were re-analysed twice to confirm the result. The ViroSeq HIV-1 Genotyping System (Celera Diagnostics, Alameda, CA) was used to genotype and to identify mutations.

\subsection{Prediction of susceptibility and subtype analysis}

The Stanford HIVdb drug resistance algorithm v.8.1.1 was applied to assess the possible impact of TDRMs on the response to therapy [20]. This algorithm assigns a drug-specific score to each TDRM detected. The score obtained from the combination of all of the TDRMs observed in a single viral strain is translated into one of five levels of susceptibility: susceptible (S); potential low-level resistance (PLR); low-level resistance (LR); intermediate resistance (IR); and high-level resistance (HR). Reduced susceptibility was scored when the Stanford system indicated the presence of resistance with at least grade LR, allowing only clinically relevant patterns to be considered. Subtyping was performed using automated subtyping of HIV-1 genetic sequences for clinical and surveillance purposes (REGA Institute Subtyping tool v.2.0 (http:// www.bioafrica.net/subtypetool/html/)) [21-23].

\subsection{Statistical analysis}

Categorical variables are expressed as percentages, and quantitative variables are expressed as the mean and standard deviation. The Chi-squared test and Fisher's exact test were applied to compare proportions, as appropriate. Student's t-test was used 
for quantitative variables. The analysis was performed with MedCalc Statistical Software version 16.8.4 (MedCalc Software bvba, Ostend, Belgium; https://www.medcalc.org; 2016). A p value of $<0.05$ was considered statistically significant.

\section{Results}

\subsection{Patients}

A valid genotyping was obtained in plasma from 750 out of 762 patients (98.4\%): their demographic characteristics and HIV subtype are reported in Table 1.

The 12 patients with a result obtained from PBMC because of failed plasma sequencing had a wild type virus.

Subtype B was the most prevalent HIV clade (505 patients, $67.3 \%)$ : non-B subtype strains were found in $19.1 \%$ of Italian patients (103 subjects) and in $67.3 \%$ of foreign-born subjects (142 subjects) $(\mathrm{p}<0.0001)$. Non-B strains included different clades: subtype $\mathrm{F}$ was the most prevalent strain described in Italian patients $(27.2 \%$ versus $8.5 \%$ in foreigns, $p=0.0001)$. The second most prevalent in Italian subjects was subtype CRF02_AG (24.3\%): this subtype was the first detected in foreign patients ( $46.5 \%, p=0.0004$ respect to Italians) (Fig. 1 ).

Overall, the diagnosis of primary/recent infection was made in 73 patients (9.7\%), a lower percentage respect to 2012-2013 $(p=0.02)$, due solely to the decrease in the cohort of patients with $B$ subtype infection $(\mathrm{p}=0.0044)$. A detailed description of patients by diagnosis and subtype is reported in Table 2.

In 2013-2016 study period subjects with chronic non-B infection were younger and with a lower CD4+ cell count and percentage respect to subjects with chronic subtype B infection ( $\mathrm{p}=0.0027, \mathrm{p}=0.0356$ and $\mathrm{p}=0.0144$ respectively).

\subsection{Susceptibility prediction}

A total of 92 patients (12.3\%) were expected to be resistant to at least one drug in the study period 2013-2016, most with a single class mutation (60/68-88.2\% in subtype B infected subjects and 23 / 24-95.8\% in non-B subjects) (Table 3). No significant differences were observed between the prevalence rates of TDRMs involving one or more drugs, except for higher rates of TDRMs that involved NNRTI as single class in subjects with non-B infection (6.9\% versus
Table 1

Distribution by subtype, ethnicity and gender of the patients enrolled from January 1, 2013 to November 16, 2016 divided by subtype B and non-B infection. The results were expressed as absolute numbers and percentages.

\begin{tabular}{lll}
\hline Enrollment periods & $2013-2016$ & \\
Total pts (n) & 750 & \\
Pts with B subtype, n (\%) & $505(67.3 \%)$ & \\
& Italian & Foreign \\
Male (n) & 381 & 51 \\
Female (n) & 55 & 18 \\
Total (n) & 436 & 69 \\
& & \\
Pts with non-B subtype, n (\%) & & \\
& $245(32.7 \%)$ & Foreign \\
Male (n) & Italian & 67 \\
Female (n) & 88 & 75 \\
Total (n) & 15 & 142 \\
$\%^{\mathrm{c}}$ & 103 & \\
$\%^{\mathrm{d}}$ & $42 \%$ & \\
\hline
\end{tabular}

Pts: patients.

a Percentage of subjects with HIV-1 B subtype infection amongst the total number of patients.

${ }^{b}$ Percentage of subjects with HIV-1 non-B subtype infection amongst the total number of patients.

c Percentage of Italian subjects amongst the total number of patients with non-B subtype.

d Percentage of Italian subjects with non-B subtype.

$3 \%, \mathrm{p}=0.0191)$ if $\mathrm{E} 138 \mathrm{~A}$ are not considered. The presence of mutation E138A (mostly reported in B patients) was not included in our previous DRM evaluation. This mutation was subsequently reported to decrease susceptibility to rilpivirine and etravirine; E138A increased, almost always detected without any other TDRM, from $2.3 \%$ in $2004-2006$ to $1.8 \%$ in $2007-2009$, to $3.2 \%$ in $2010-$ 2012 and to 3.6\% over the latter time period. In 2013-2016 E138A was found in 26 B subtype (5.1\%) and in only two (0.8\%) non-B infected subjects (Table 3 ).

Other nine out of 48 B subtype (19\%, 1.8\% of the cohort) and 5 out of twenty non B subtype patients ( $25 \%, 2 \%$ of the cohort) with resistance to NNRTI-class, revealed TRDMs such as 101E, 138K, 181C and 190E, which compromise also new agents available for first line therapy.

This data was confirmed also as relative weight amongst the total resistant strains as single class ( $82.6 \%$ in patients with non-B versus $68.3 \%$ with B subtype infection) and as global prevalence including also combinations (83.3\% versus $70.6 \%$ ).

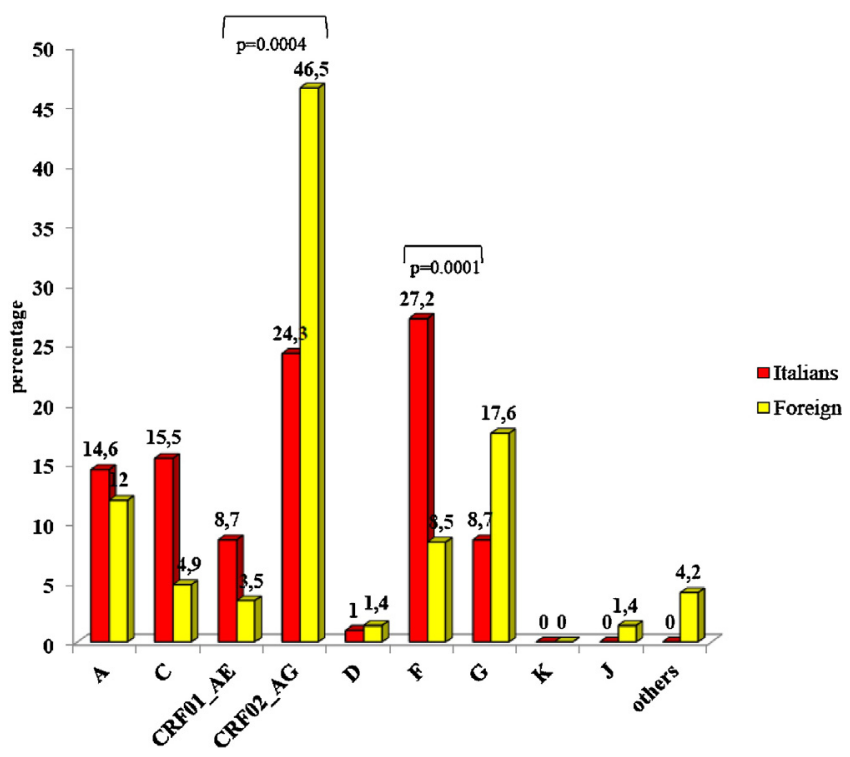

Fig. 1. Distribution of non-B subtype clades identified in Italian patients and foreign subjects. Data are expressed as relative percentage. 
Table 2

Age, CD4+ cell count and percentage amongst primary-recent and chronic HIV infections of the patients enrolled from January 1, 2013 to November 16, 2016. Patients are divided by subtype B and non-B subtype infection. Quantitative values are expressed as mean and standard deviation.

\begin{tabular}{lll}
\hline & $2013-2016$ & \\
B subtype & 505 & \\
Diagnosis & Primary & Chronic \\
Pts n (\%) & $49(9.7)$ & $456(90.3)$ \\
Age (years) & $34.3(11.2)$ & $40.4(11.3)$ \\
CD4+ cells ${ }^{\mathrm{a}}$ & $512(263)$ & $394(271)$ \\
CD4+ cells \% & $27.1(10.6)$ & $20.8(11.1)$ \\
& & \\
Non-B subtype & 245 & Chronic \\
Diagnosis & Primary & $221(90.2)$ \\
Pts n (\%) & $24(9.8)$ & $37.6(11.5)$ \\
Age (years) & $33.4(9.4)$ & $347(275)$ \\
CD4+ cells & $562(241)$ & $18.5(12.1)$ \\
CD4\% & $27.7(8.5)$ & \\
\hline
\end{tabular}

Pts: patients.

a Cells $/ \mathrm{mm}^{3}$.

Conversely, NRTI-related TDRM weight was higher in patients with subtype B virus both as single class (28.3\% versus $13 \%)$ and as total frequency (33.8\% versus $16.7 \%$ ).

Overall, in 2013-2016 the prevalence of TDRMs increased in subjects with non-B infection respect to 2010-2012: resistance rates for NRTI, NNRTI and PI are comparable in the two study periods. The detailed description is reported in Table 3. Amongst non-B subtypes, NNRTI-related DRMs were found in eleven foreign patients (only one in a case of acute-recent infection and one, in combination with NRTI-DRM, in a chronic infected) and they were mainly $103 \mathrm{~N}$ or $181 \mathrm{C}$. Only seven Italian patients with non-B chronic infection had NNRTI-DRM, and they were mainly $103 \mathrm{~N}$ or $181 \mathrm{C}$ as well.

Patients with primary-recent infection had almost only TDRMs involving a single class in 2013-2016 study period: the frequency was lower respect to the study interval 2010-2012 (Table 4).

\subsection{Susceptibility prediction to integrase strand transfer inhibitors}

Sequences of integrase region were performed in 484 out of 750 subiects enrolled from 1 January 2013, according to availability of plasma samples. No selection criteria was applied; 40 samples were collected in 2013, 163 in 2014, 155 in 2015, 126 in 2016 (Tables 5a and 5b).

Amongst the 361 patients infected with B subtype, 43 (11.9\%) had a primary-recent infection and only one diagnosed in $2013 \mathrm{had}$ a $157 \mathrm{Q}$ mutation, a polymorphic accessory mutation. This subject had no other TDRMs. Three hundred and eighteen patients with chronic infection and B subtype demonstrated a wild type virus in 277 cases (87.7\%); 32 (10.1\%) had resistance to one or more of NRTI, NNRTI or PI and 7 (2.2\%) had 157Q (in two subjects associated to NNRTI). Two $157 \mathrm{Q}$ were detected in 2013, 2 in 2014, one in 2015 and 2 in 2016. G163RK, non-polymorphic mutations in all subtypes except subtype F, were recovered in two patients, G163K in 2015 and G163R in 2016.

Amongst the 123 patients infected with non-B subtype, 10 (8.1\%) had a primary-recent infection and none had InSTI related TDRM. Ninety-nine chronic subjects with non-B subtypes revealed a wild type (80.5\%), 8 (6.5\%) had resistance to one or more of NRTI, NNRTI or PI and five had 157Q (in one subject associated to NNRTI). One $157 \mathrm{Q}$ were detected in 2013, two in 2014, one in 2015 and one in 2016. One patient diagnosed in 2016 had 143C, a non-polymorphic major mutation; this subject had no other TDRMs.

Of note, in the eight years period from 2009 to 2016, in the seven infectious disease units of the Veneto area contributing to this work, 83 experienced and InSTI failed patients were found to have InSTI major DRMs by analysis in our laboratory: two in 2009, four in 2010, eight in 2011, two in 2012, fourteen in 2013, eleven in 2014, sixteen in 2015 and twenty-six in 2016. About 4000 patients were on treatment in these units, with viremic and failing subjects able to transmit a resistant virus.

Table 3

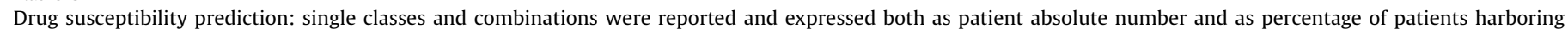
transmitted drug resistance mutations.

\begin{tabular}{|c|c|c|c|c|c|c|c|}
\hline $\begin{array}{l}\mathrm{NRTI}^{\mathrm{a}} \\
\mathrm{n}(\%)\end{array}$ & $\begin{array}{l}\mathrm{NNRTI}^{\mathrm{b}} \\
\mathrm{n}(\%)\end{array}$ & $\begin{array}{l}\mathrm{PI}^{\mathrm{C}} \\
\mathrm{n}(\%)\end{array}$ & $\begin{array}{l}\text { NRTI + NNRTI } \\
\mathrm{n}(\%)\end{array}$ & $\begin{array}{l}\text { NRTI + PI } \\
\mathrm{n}(\%)\end{array}$ & $\begin{array}{l}\text { NNRTI + PI } \\
\mathrm{n}(\%)\end{array}$ & $\begin{array}{l}3 \text { drug classes } \\
\mathrm{n}(\%)\end{array}$ & $\begin{array}{l}\text { Total } \\
\mathrm{n}(\%)\end{array}$ \\
\hline $\begin{array}{l}\text { B subtype } \\
17(3.3 \%)\end{array}$ & $\begin{array}{l}15(3 \%) \\
41^{\mathrm{d}}(8.1 \%)^{\mathrm{d}}\end{array}$ & $2(0.4 \%)$ & $4(0.8 \%)$ & $1(0.2 \%)$ & $2(0.4 \%)$ & $1(0.2 \%)$ & $\begin{array}{l}42 / 505(8.3 \%) \\
68 / 505^{\mathrm{d}}(13.5 \%)^{\mathrm{d}}\end{array}$ \\
\hline $\begin{array}{l}\text { Non-B sub } \\
3(1.2 \%)\end{array}$ & $\begin{array}{l}17(6.9 \%) \\
19^{\mathrm{d}}(7.7 \%)^{\mathrm{d}}\end{array}$ & $1(0.4 \%)$ & $1(0.4 \%)$ & 0 & 0 & 0 & $\begin{array}{l}22 / 245(9 \%) \\
24 / 245^{\mathrm{d}}(9.8 \%)^{\mathrm{d}}\end{array}$ \\
\hline
\end{tabular}

a NRTI: nucleoside Reverse transcriptase inhibitors.

b NNRTI: non-nucleoside reverse transcriptase inhibitors.

c PI: protease inhibitors.

d Including 138A.

Table 4

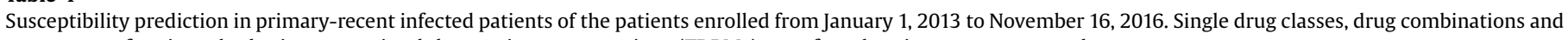
percentage of patients harboring transmitted drug resistance mutations (TDRMs) out of total patients were reported.

\begin{tabular}{|c|c|c|c|c|c|c|c|c|}
\hline & NRTI & NNRTI & PI & NRTI + NNRTI & $\mathrm{NRTI}+\mathrm{PI}$ & NNRTI + PI & Three classes & Total TDRMs/total patients \\
\hline \multicolumn{9}{|c|}{ B subtype } \\
\hline $2013-16$ & 1 & 1 & 0 & 1 & 0 & 0 & 0 & $3 / 49(6.1 \%)$ \\
\hline \multicolumn{9}{|c|}{ Non-B subtype } \\
\hline $2013-16$ & 0 & 1 & 0 & 0 & 0 & 0 & 0 & $1 / 24(4.1 \%)$ \\
\hline
\end{tabular}

NRTI: nucleoside reverse transcriptase inhibitors.

NNRTI: non-nucleoside reverse transcriptase inhibitors.

PI: protease inhibitors. 
Table 5a

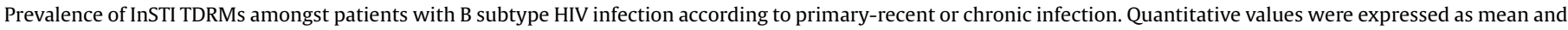
standard deviation.

\begin{tabular}{|c|c|c|c|}
\hline & All patients & Pts with primary-recent infection & Pts with chronic infection \\
\hline Number & 361 & 43 & 318 \\
\hline Age (years) & $38.8(10.8)$ & $32(8.3)$ & $39.7(10.8)$ \\
\hline CD4+ cell count ${ }^{\mathrm{a}}$ & $401(273)$ & $501(265)$ & $386(271)$ \\
\hline CD4+ cells \% & $20.8(10.9)$ & $25.5(9.3)$ & $20.2(10.9)$ \\
\hline Plasma HIV RNA ${ }^{\mathrm{b}}$ & $501,853(1,594,266)$ & $1,346,175(2,783,875)$ & $389,049(1,328,951)$ \\
\hline Pts with wild type virus, $\mathrm{n}(\%)$ & $316(87.5)$ & $39(90.7)$ & $277(87.1)$ \\
\hline Pts with NRTI TDRMs, n (\%) & $14(3.9)$ & $1(2.3)$ & $13(4.1)$ \\
\hline Pts with NNRTI TDRMs, n (\%) & $15(4.1)$ & $1(2.3)$ & $14(4.4)$ \\
\hline Pts with PI TDRMs, n (\%) & $1(0.3)$ & 0 & $1(0.3)$ \\
\hline Pts with NRTI + NNRTI TDRMs, n (\%) & $4(1.1)$ & $1(2.3)$ & $3(0.9)$ \\
\hline Pts with NRTI + PI TDRMs, n (\%) & $1(0.3)$ & 0 & $1(0.3)$ \\
\hline Pts with NRTI + NNRTI + PI TDRMs, n (\%) & $1(0.3)$ & 0 & $1(0.3)$ \\
\hline Pts with $157 \mathrm{Q}$ mutation, $\mathrm{n}(\%)$ & $8(2+\mathrm{NNRTI})(2.2)$ & $1(2.3)$ & $7(2+\mathrm{NNRTI})(2.2)$ \\
\hline Pts with $163 \mathrm{R} / \mathrm{K}$ mutation, n (\%) & $2(0.6)$ & 0 & $2(0.6)$ \\
\hline Pts with 68IV mutation, $\mathrm{n}(\%)$ & $1(0.3)$ & 0 & $1(0.3)$ \\
\hline Pts with 97A mutation, $\mathrm{n}(\%)$ & $1(0.3)$ & 0 & $1(0.3)$ \\
\hline Pts with74IM mutation, $\mathrm{n}(\%)$ & $6(1.7)$ & 0 & $6(1.9)$ \\
\hline Pts with119R mutation, n (\%) & $2(0.6)$ & 0 & $2(0.6)$ \\
\hline Pts with 121 CFSY mutation, n (\%) & $1(0.3)$ & 0 & $1(0.3)$ \\
\hline Pts with 151I mutation, $\mathrm{n}(\%)$ & $1(0.3)$ & $1(2.3)$ & 0 \\
\hline Pts with 163KRT mutation, n (\%) & $2(0.6)$ & 0 & $2(0.6)$ \\
\hline
\end{tabular}

InSTI: integrase strand-transfer inhibitors.

NRTI: nucleoside reverse transcriptase inhibitors.

NNRTI: non-nucleoside reverse transcriptase inhibitors.

PI: protease inhibitors.

Pts: patients.

TDRMs: transmitted drug resistance mutations.

a Cells $/ \mathrm{mm}^{3}$.

b Copies/ml.

Table 5b

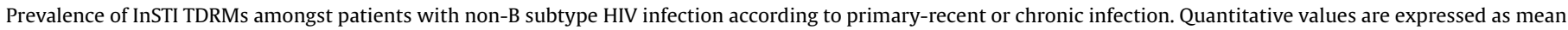
and standard deviation.

\begin{tabular}{|c|c|c|c|}
\hline & All patients & Pts with primary-recent infection & Pts with chronic infection \\
\hline Number & 123 & 10 & 113 \\
\hline Age (years) & $36.8(11)$ & $31.6(3.3)$ & $37.3(11)$ \\
\hline CD4+ cell count ${ }^{\mathrm{a}}$ & $383(286)$ & $543(197)$ & $369(285)$ \\
\hline CD4+ cells \% & $19(11.4)$ & $23.3(8.7)$ & $18.3(11.4)$ \\
\hline Plasma HIV RNA ${ }^{\mathrm{b}}$ & $718,784(2,045,027)$ & $1,056,657(2,663,855)$ & $684,130(1,996,636)$ \\
\hline Pts with wild type virus, $\mathrm{n}(\%)$ & $109(88.6)$ & $10(100)$ & $99(87.6)$ \\
\hline Pts with NRTI TDRMs, n (\%) & $2(1.6)$ & 0 & $2(1.8)$ \\
\hline Pts with NNRTI TDRMs, n (\%) & $6(4.9)$ & 0 & $6(5.3)$ \\
\hline Pts with PI TDRMs, n (\%) & $1(0.8)$ & 0 & $1(0.9)$ \\
\hline Pts with NRTI + NNRTI TDRMs, n (\%) & 0 & 0 & 0 \\
\hline Pts with NRTI + PI TDRMs, $\mathrm{n}(\%)$ & 0 & 0 & 0 \\
\hline Pts with NRTI + NNRTI + PI TDRMs, n (\%) & 0 & 0 & 0 \\
\hline Pts with $157 \mathrm{Q}$ mutation, $\mathrm{n}(\%)$ & $5(1+\mathrm{NNRTI})(4.1)$ & 0 & $5(1+\mathrm{NNRTI})(4.4)$ \\
\hline Pts with $143 \mathrm{C}$ mutation, n (\%) & $1(0.8)$ & & $1(0.9)$ \\
\hline Pts with 68IV mutation, n (\%) & $1(0.8)$ & 0 & $1(0.9)$ \\
\hline Pts with 97A mutation, $\mathrm{n}(\%)$ & $20(1+P I)(16.3)$ & 0 & $20(1+P I)(17.7)$ \\
\hline Pts with 74IM mutation, n (\%) & $20(1+P I)(16.3)$ & $1(10)$ & $19(1+\mathrm{PI})(16.8)$ \\
\hline Pts with 119R mutation, n (\%) & $2(1.6)$ & $1(10)$ & $1(0.9)$ \\
\hline Pts with 121 CFSY mutation, $\mathrm{n}(\%)$ & 0 & 0 & 0 \\
\hline Pts with 151I mutation, $\mathrm{n}(\%)$ & 0 & 0 & 0 \\
\hline Pts with 260 I mutation, $\mathrm{n}(\%)$ & $1(0.8)$ & 0 & $1(0.9)$ \\
\hline
\end{tabular}

InSTI: integrase strand-transfer inhibitors.

NRTI: nucleoside reverse transcriptase inhibitors.

NNRTI: non-nucleoside reverse transcriptase inhibitors.

PI: protease inhibitors.

Pts: patients.

TDRMs: transmitted drug resistance mutations.

a Cells $/ \mathrm{mm}^{3}$.

b Copies/ml. 


\section{Discussion}

This study described TDRMs identified in ART naive subjects during the last four years in a well identified geographical setting with a known and previously analysed HIV patients cohort (years 2004-2012) [14]. This characteristic allowed to show a timely picture and to point out how TDRMs modified with the progressive increase of the InSTI inclusion in the ART regimens.

Our data on higher frequency of TDRMs in patients with $B$ subtype respect to those with non-B infection when E138A is included in the analysis are in agreement with those reported by Descamps et al. [24] in a $2010-2011$ survey (11.9\% versus $5.1 \%$ respectively). Conversely, we reported a comparable rate of TDRMs in the two cohort of our patients (8.3\% and 9\% respectively) when E138A is not evaluated, because of the higher frequency of detection of E138A in patients with subtype B infection.

The decrease of TDRMs identified in patients with primary infection was reported both in subjects with non-B and B infection but the number of patients with primary infection was significantly lower respect to 2010-2012 only in the cohort of individuals infected with subtype B. There is a great variability in TDRMs frequency described in patients diagnosed with acute disease and different inclusion criteria may play a role [12,25,26]. Resistance mutations that affect replication may revert and outgrowth of wild type virus, which is more replication competent, may occur in patients infected for less than 6 months [27]. Furthermore, transmitted viruses that contain mutations may be archived in lymphocyte reservoirs very early during acute HIV infection [4].

Mutations associated with NNRTI alone were observed frequently, $3 \%$ insubtype $\mathrm{B}$ and $6.9 \%$ in non-B subtype virus infection. Of note, these frequencies raised up to $8.1 \%$ and $7.7 \%$ respectively when polymorphic mutation E138A is included and to 9.5\% and to 8.2 when all NNRTI combinations are considered. E138A mutation (always as isolated mutation) was detected mostly in patients with subtype B, who are the $67.3 \%$ of our study population, with a prevalence comparable to that described by Alvarez et al. [28] in a Spanish cohort of naive patients recruited in the period 2007-2011: however in this study no data on patient's HIV genotype was included. Analysis by genotype was available in a French study including patients tested from 2008 to 2011: only half of patients had a B subtype virus and E138A was found in $2 \%$ of them, versus $4.1 \%$ in non-B subtype viruses [29]. It was not possible to speculate on the origin of the acquisition of resistant strains in non-B subtypes; our analysis was performed at the time of HIV diagnosis, but most TDRMs in non-B subtypes were found in subjects with chronic infection and were NNRTI-related DRMs.

Ripilvirine became more widely used in clinical practice as third drug alternative to InSti in selected clinical conditions [2]: of note, a small proportion of subjects with NNRTI-TDRM was predicted to fail also the only NNRTI-recommended first line regimen, then resistance testing at beginning is mandatory.

Amongst non-B subtypes, CRF02_AG was the most prevalent strain detected in foreign patients and the second most frequent and in Italians this spread had clinical implications, because of possible under-quantification of plasma HIV RNA value with commercial tests [30-32].

Interestingly, the non-B subtype pattern is different in Italians and foreigns, suggesting a compartmentalised transmission in newly HIV diagnosed Italian patients. Subtype F was identified in a growing proportion of subjects with non-B virus [14,33]: Pernas et al. [34] reported that subtype F was an independent predictor of poor virological response after 24 weeks of ART in patients at the first HIV diagnosis from 2009 to 2013 (the majority being Spanish).

No signature InSTI-resistant variants were circulating in Europe before the introduction of InSTIs and more recently polymorphisms and substitutions conferring low-level resistance to raltegravir and elvitegravir were found in acute-recent infected patients $[16,19]$. Prevalence of InSTI transmitted drug resistance may increase with the increasing use of InSTIs, but it was recently reported that their prevalence was stable although InSTIs were increasingly used in the Swiss cohort [18], and this is in contrast to the introduction of previous drug classes where more treatment failures with resistant strains occurred and TDR was observed more rapidly. Likewise, the wide availability of InSTIs from at least seven years in our hospitals allowed to determine the conditions to select resistant strains and to transmit those strains to the newly infected or to superinfect HIV-patients. Despite the detection of a substantial number of resistant strains in the treated population, we demonstrate on a considerable population sample recruited from 2013 until very recent times, including $11 \%$ of recently infected, the almost total absence of major InSTI-TDRM (one out of 484 subjects) in both chronic and primary-recent infected with B and non-B subtypes in our region. Few polymorphic accessory variants were detected, quite always as isolated mutations. Of note, we identified $263 \mathrm{~K}$ mutation in a patient with subtype B infection: it was recently detected only by ultradeep sequencing in two out of 92 naive patients newly diagnosed in 2013-2015 [35]. Monitoring of TDRMs should continue in the light of persisting presence of NNRTI-related mutations amongst newly diagnosed subjects, sometime impacting also to modern NNRTI drugs recommended in first-line therapy; further a surveillance of InSTI-TDRMs should be performed given the role of these drugs in recent guidelines.

\section{Funding}

This work was supported by PRIN 2012 to SGP and by MURST ex 60\% (60A07-2972/15) to MB.

\section{Competing interests}

None declared.

\section{Ethical approval}

The study was approved by the Ethical Committee for Clinical Experimentation, Padua Province (Ethics Committee Protocol no. 2606-12P). The subjects gave informed consent for all procedures and for the use of their blinded data for scientific evaluation and publication. This study was conducted in accordance with the Helsinki Declaration and local legislation.

\section{Acknowledgements}

Preliminary data from this study were presented as an oral communication at the 55th Interscience Conference on Antimicrobial Agents and Chemotherapy (ICAAC), 17-21 September, 2015, San Diego.

MB, RS, MC, RF, VM, SP and SGP are members of CAVeAT (Cohort of Amici Venetians for Antiretroviral Treatment).

\section{References}

[1] Günthard HF, Saag MS, Benson CA, del Rio C, Eron JJ, Gallant JE, et al. Antiretroviral drugs for treatment and prevention of HIV infection in adults: 2016 Recommendations of the International Antiviral Society-USA Panel. JAMA 2016;316:191-210.

[2] European AIDS Clinical Society EACS guidelines version 8.0. Available at: http://www.eacsociety.org/files/guidelines_8_0-english_web.pdf. [Accessed 29 October 2015].

[3] Parisi SG, Boldrin C, Cruciani M, Nicolini G, Cerbaro I, Manfrin V, et al. Both human immunodeficiency virus cellular DNA sequencing and plasma RNA sequencing are useful for detection of drug resistance mutations in blood samples from antiretroviral-drug-naive patients. J Clin Microbiol 2007;45:1783-8. 
[4] Parisi SG, Mazzi R, Boldrin C, Dal Bello F, Franchin E, Andreoni M, et al. Drug resistance mutations can be archived very early in HIV primary infection. AIDS 2006;20:1337-8.

[5] Baxter JD, Dunn D, White E, Sharma S, Geretti AM, Kozal MJ, et al. Global HIV1 transmitted drug resistance in the INSIGHT Strategic Timing of AntiRetroviral Treatment (START) trial. HIV Med 2015;16(Suppl. 1):77-87.

[6] Pham QD, Wilson DP, Law MG, Kelleher AD, Zhang L. Global burden of transmitted HIV drug resistance and HIV-exposure categories: a systematic review and meta-analysis. AIDS 2014;28:2751-62.

[7] Buchacz K, Young B, Palella Jr. FJ, Armon C, Brooks JT. Trends in use of genotypic resistance testing and frequency of major drug resistance among antiretroviral-naive persons in the HIV Outpatient Study, 1999-2011. J Antimicrob Chemother 2015;70:2337-46.

[8] Vercauteren J, Wensing AM, van de Vijver DA, Albert J, Balotta C, Hamouda O, et al. Transmission of drug-resistant HIV-1 is stabilizing in Europe. J Infect Dis 2009;200:1503-8.

[9] Hofstra LM, Sauvageot N, Albert J, Alexiev I, Garcia F, Struck D, et al. Transmission of HIV drug resistance and the predicted effect on current firstline regimens in Europe. Clin Infect Dis 2016;62:655-63.

[10] Rhee SY, Blanco JL, Jordan MR, Taylor J, Lemey P, Varghese V, et al. Geographic and temporal trends in the molecular epidemiology and genetic mechanisms of transmitted HIV-1 drug resistance: an individual-patient- and sequencelevel meta-analysis. PLoS Med 2015;12:e1001810.

[11] Panichsillapakit T, Smith DM, Wertheim JO, Richman DD, Little SJ, Mehta SR. Prevalence of transmitted HIV drug resistance among recently infected persons in San Diego, CA 1996-2013. J Acquir Immune Defic Syndr 2016;71:228-36.

[12] Ambrosioni J, Sued O, Nicolas D, Parera M, López-Diéguez M, Romero A, et al. Trends in transmission of drug resistance and prevalence of non-B subtypes in patients with acute or recent HIV-1 infection in Barcelona in the last 16 years (1997-2012). PLoS One 2015;10:e0125837.

[13] Frange P, Assoumou L, Descamps D, Chéret A, Goujard C, Tran L, et al. HIV1 subtype B-infected MSM may have driven the spread of transmitted resistant strains in France in 2007-12: impact on susceptibility to first-line strategies. J Antimicrob Chemother 2015;70:2084-9.

[14] Parisi SG, Andreis S, Scaggiante R, Cruciani M, Ferretto R, Manfrin V, et al. Decreasing trends of drug resistance and increase of non-B subtypes amongst subjects recently diagnosed as HIV-infected over the period 2004-2012 in the Veneto Region, Italy. J Glob Antimicrob Resist 2013:1:201-6.

[15] Montagna C, Mazzuti L, Falasca F, Maida P, Bucci M, D'Ettorre G, et al. Trends in drug resistance-associated mutations in a real-life cohort of Italian patients infected with HIV-1. J Glob Antimicrob Resist 2015;3:267-72.

[16] Casadellà M, van Ham PM, Noguera-Julian M, van Kessel A, Pou C, Hofstra LM, et al. Primary resistance to integrase strand-transfer inhibitors in Europe. J Antimicrob Chemother 2015;70:2885-8.

[17] Doyle T, Dunn DT, Ceccherini-Silberstein F, De Mendoza C, Garcia F, Smit E et al. Integrase inhibitor (INI) genotypic resistance in treatment-naive and raltegravir-experienced patients infected with diverse HIV-1 clades. J Antimicrob Chemother 2015;70:3080-6.

[18] Scherrer AU, Yang WL, Kouyos RD, Böni J, Yerly S, Klimkait T, et al. Successful prevention of transmission of integrase resistance in the Swiss HIV Cohort Study. J Infect Dis 2016;214:399-402.

[19] Ambrosioni J, Nicolás D, Manzardo C, Agüero F, Blanco JL, Mosquera MM, et al. Integrase strand-transfer inhibitor polymorphic and accessory resistance substitutions in patients with acute/recent HIV infection. J Antimicrob Chemother 2017;72:205-9.

[20] Stanford HIV drug resistance database. http://hivdb.stanford.edu. [Accessed 20 November 2016].

[21] Pineda-Peña AC, Faria NR, Imbrechts S, Libin P, Abecasis AB, Deforche K, et al. Automated subtyping of HIV-1 genetic sequences for clinical and surveillance purposes: performance evaluation of the new REGA version 3 and seven other tools. Infect Genet Evol 2013;19:337-48.

[22] Alcantara LCJ, Cassol S, Libin P, Deforche K, Pybus OG, Van Ranst M, et al. A standardized framework for accurate, high-throughput genotyping of recombinant and non-recombinant viral sequences. Nucleic Acids Res 2009;37(Web Server issue):W634-42.

[23] de Oliveira T, Deforche K, Cassol S, Salminem M, Paraskevis D, Seebregts C, et al. An automated genotyping system for analysis of HIV-1 and other microbial sequences. Bioinfomatics 2005;21:3797-800.

[24] Descamps D, Assoumou L, Chaix ML, Chaillon A, Pakianather S, de Rougemont A, et al. National sentinel surveillance of transmitted drug resistance in antiretroviral-naive chronically HIV-infected patients in France over a decade: 2001-2011. J Antimicrob Chemother 2013;68:2626-31.

[25] Booth CL, Geretti AM. Prevalence and determinants of transmitted antiretroviral drug resistance in HIV-1 infection. J Antimicrob Chemother 2007;59:1047-56.

[26] Yanik EL, Napravnik S, Hurt CB, Dennis A, Quinlivan EB, Sebastian J, et al. Prevalence of transmitted antiretroviral drug resistance differs between acutely and chronically HIV-infected patients. J Acquir Immune Defic Syndr 2012;61:258-62.

[27] Wainberg MA, Moisi D, Oliveira M, Toni TD, Brenner BG. Transmission dynamics of the M184V drug resistance mutation in primary HIV infection. J Antimicrob Chemother 2011;66:2346-9.

[28] Alvarez M, Monge S, Chueca N, Guillot V, Viciana P, Anta L, et al. Transmitted drug resistance to rilpivirine in newly diagnosed antiretroviral naive adults. Clin Microbiol Infect 2015;21(104):e1-5.

[29] Lambert-Niclot S, Charpentier C, Storto A, Fofana DB, Soulié C, Fourati S, et al Prevalence of pre-existing resistance-associated mutations to rilpivirine, emtricitabine and tenofovir in antiretroviral-naive patients infected with B and non-B subtype HIV-1 viruses. J Antimicrob Chemother 2013;68:1237-42.

[30] Lai A, Riva C, Marconi A, Balestrieri M, Razzolini F, Meini G, et al. Changing patterns in HIV-1 non-B clade prevalence and diversity in Italy over three decades. HIV Med 2010;11:593-602.

[31] Ndiaye O, Diop-Ndiaye H, Ouedraogo AS, Fall-Malick FZ, Sow-Sall A, Thiam M et al. Comparison of four commercial viral load techniques in an area of non-B HIV-1 subtypes circulation. J Virol Methods 2015;222:122-31.

[32] Tatarelli P, Taramasso L, Di Biagio A, Sticchi L, Nigro N, Barresi R, et al. HIV 1 RNA quantification in CRF02_AG HIV-1 infection: too easy to make mistakes. New Microbiol 2016;39:150-2.

[33] Torti C, Lapadula G, Izzo I, Brindicci G, Labbate G, Quiros-Roldan E, et al Heterogeneity and penetration of HIV-1 non-subtype B viruses in an Italian province: public health implications. Epidemiol Infect 2010;138:1298-307.

[34] Pernas B, Grandal M, Mena A, Castro-Iglesias A, Cañizares A, Wyles DL, et al. High prevalence of subtype $\mathrm{F}$ in newly diagnosed HIV-1 persons in northwest Spain and evidence for impaired treatment response. AIDS 2014;28:1837-40.

[35] Jaffré J, Armenia D, Bellocchi MC, Wirden M, Carioti L, Lambert S, et al. Ultradeep sequencing detection of the R263K integrase inhibitor drug resistance mutation. J Antimicrob Chemother 2017;72:1537-9. 